\title{
SUBSETS AND SUBGRAPHS WITH MAXIMAL PROPERTIES ${ }^{1}$
}

\author{
OYSTEIN ORE AND T. S. MOTZKIN ${ }^{2}$
}

1. Introduction. One is often concerned with subsets $H$ of a set $G$ such that $H$ is maximal among all those subsets of $G$ which have a certain property $P$. This property $P$ usually takes the form that a family

$$
F_{1}, F_{2}, \cdots
$$

of special subsets shall be excluded or forbidden in $H$. These subsets (1.1) shall remain fixed in the following. To avoid trivial cases we assume that the void subset is not admitted in (1.1).

A subset $H$ of $G$ not containing any of the sets (1.1) as subsets shall be called an exclusion set. $H$ is a maximal (exclusion) set when it is not contained in any greater exclusion set. The object of the present paper is to investigate certain special properties of such maximal sets, with applications to maximal graphs.

It shall be assumed always that (1.1) represents a finite type exclusion, that is, all sets $F$ shall be finite. From the maximal principle $[1$, p. 132] we conclude immediately

TheOREM 1.1. Every exclusion set is contained in a maximal exclusion set.

2. Maximal graphs. We observe that, if $G$ is a graph (set of edges, i.e., vertex pairs), one can also establish the existence of maximal graphs $H$ excluding the graphs (1.1) and having restricted local degrees

$$
\rho_{H}(v) \leqq \kappa(v)
$$

where the constants $\kappa(v)$ are prescribed for each vertex $v$. For this purpose it is sufficient to adjoin to the subgraphs (1.1) all star graphs consisting of $k(v)+1$ edges having the endpoint $v$.

From the properties of the excluded subgraphs (1.1) one can draw various conclusions in regard to the properties of the corresponding maximal graphs. We say that a subgraph $H$ covers $G$ when there is an

Received by the editors December 1, 1958 and, in revised form, March 14, 1959. tion.

1 This research has been supported by a grant from the National Science Founda-

2 The paper is written by Oystein Ore with a note at the end by T. S. Motzkin. 
edge of $H$ at every (nonisolated) vertex of $G$. A terminal edge is an edge which is the only edge at one of its ends.

THEOREM 2.1. If none of the excluded graphs has terminal edges then any maximal graph covers $G$.

Proof. If in some $H$ there should be no edges at a vertex $v$ of $G$ then any edge from $v$ could be added to $H$ without producing a forbidden subgraph.

This result can be extended as follows: Let $\mu>0$ be the minimum of the local degrees of the graphs (1.1). Then at any vertex $v$ a maximal graph $H$ must have at least

$$
\min (\mu-1, \rho(v))
$$

edges where $\rho(v)$ is the local degree at $G$ at $v$. When $v$ is not a vertex in any $F$ then

$$
\rho_{H}(v)=\rho(v) .
$$

When $G$ is connected one may ask for conditions under which the maximal graphs are connected. Assume that none of the excluded graphs (1.1) has bridges, that is, edges appearing in no circuits. By this definition it follows that any maximal graph $H$ covers $G$. Suppose that $H$ has some connected component $H_{1}$. Since $G$ is connected there exists some edge $E$ connecting $H_{1}$ with some other component $H_{2}$. But then $E$ could be added to $H$ without producing any forbidden subgraphs since it is a bridge in $H+E$. This gives:

Theorem 2.2. Let $G$ be a connected graph and assume that none of the graphs (1.1) has bridges. Then any maximal graph $H$ is connected and covers the vertices of $G$.

When the excluded graphs are circuits these conditions are fulfilled with $\mu=2$. As an example one may take the bipartite subgraphs of $G$. These are characterized by the property that they contain no circuits with an odd number of edges (see [2, Chapter 11, \$4]). We conclude that there exist maximal bipartitite subgraphs covering the vertices of $G$ and they are connected when $G$ is connected.

One can generalize Theorem 2.2 as follows: When the graph $G$ is $t$-edge connected and each excluded graph $F$ in $(1.1)$ is $(t+1)$-edge connected then a maximal graph is $t$-edge connected.

3. Reduction sets. We return to the consideration of a general set $G$, with excluded subsets (1.1). The adjunction of a set of elements

$$
E=\sum E_{i}
$$


to $H$ will produce certain forbidden subsets $F_{j}$ in the set $H+E$. The sum set

$$
F(H+E)=\sum F_{j}
$$

we call the forbidden component of $H+E$. Each $F_{j}$ contains at least one of the elements $E_{i}$, and $F(H+E)$ contains $E$.

A set of elements in $F(H+E)$

$$
R=\sum R_{k}
$$

shall be called a reduction set for $F(H+E)$ if its complement

$$
F(H+E)-R
$$

is maximal in $F(H+E)$. The set $E$ is a trivial example of a reduction set for $F(H+E)$.

Then the set

$$
H_{1}=H+E-R
$$

is an exclusion set. It is a maximal set if and only if the adjunction of any single element $E_{0}$ to $E$ produces forbidden subsets in (3.1), that is, if $R$ is not a reduction set for any set larger than $E$.

4. Simple maximal sets. We shall apply these observations to some special cases. The maximal set $H$ shall be called simple if the adjunction of any single element $E$ to $H$ produces only a single excluded subset $F(H+E)$. Any element $R$ in $F(H+E)$ is then a reduction element and the set $H_{1}$ in (3.1) is an exclusion set which we say has been obtained from $H$ by a simple interchange.

We define further: A simple maximal set is strongly simple when it has the property that no forbidden component $F\left(H+E_{1}+E_{2}\right)$ defined by two elements can be reduced to an exclusion set by the removal of a single element. The preceding discussion shows:

TheOREM 4.1. When $H$ is a strongly simple maximal set then any simple interchange gives another maximal set.

Now take $H_{1}$ and $H_{2}$ to be two different strongly simple maximal sets and $E_{2}$ an element in $H_{2}$ not in $H_{1}$. The adjunction of $E_{2}$ to $H_{1}$ produces a single excluded set $F\left(H+E_{2}\right)$ in $H_{1}+E_{2}$. This set $F\left(H+E_{2}\right)$ cannot lie entirely in $H_{2}$ so that it contains at least one element $E_{1}$ in $H_{1}$ but not in $H_{2}$. The interchange of $E_{1}$ and $E_{2}$ gives a new maximal set $H_{1}^{\prime}$ differing from $H_{2}$ in one pair of elements less than $H_{1}$ and $H_{2}$.

From this observation one deduces if $H_{1}\left(\right.$ or $\left.H_{2}\right)$ is finite:

THEOREM 4.2. Let all maximal sets in $G$ be strongly simple; then for 
any two such sets $H_{1}$ and $H_{2}$ one can construct a one-to-one correspondence between their elements.

To see that such a correspondence exists for any two infinite strongly simple maximal sets $H_{1}$ and $H_{2}$, let $c_{0}, c_{1}, c_{2}$ be the cardinals of $H_{0}$ (the intersection of $H_{1}$ and $H_{2}$ ), $H_{1}-H_{0}, H_{2}-H_{0}$. Then to each element $E$ of $H_{2}-H_{0}$ there corresponds a different nonempty finite subset $F\left(H_{1}+E\right)-E$ of $H_{1}$; hence $c_{2} \leqq\left(c_{0}+c_{1}\right)^{2}=c_{0}+c_{1}=\max \left(c_{0}, c_{1}\right)$, and likewise $c_{1} \leqq \max \left(c_{0}, c_{2}\right)$. Both if $c_{0} \geqq \max \left(c_{1}, c_{2}\right)$ and if $c_{0}<\max \left(c_{1}, c_{2}\right)$ we obtain $c_{0}+c_{1}=c_{0}+c_{2}$, q.e.d.

We shall use the terminology that two sets are simply related if one can be transformed into the other by a series of simple interchanges. The preceding shows:

TheOREM 4.3. When a maximal set in Theorem 4.2 is finite then all such sets are simply related, hence have the same number of elements.

5. Maximal trees. As an example for this discussion let us consider the case where the family (1.1) of excluded subsets consists of all circuits in a graph $G$. It follows immediately that $G$ has maximal circuitless graphs $T$ which cover all vertices of $G$. If $G$ is connected the graph $T$ is a tree (see König [2, Chapter 4]).

Let us add an edge $E$ to such a maximal tree $T$. Then $T+E$ contains a single circuit since the two end vertices of $E$ are connected by a single path in $T$. Thus $T$ is simple. If two edges $E_{1}$ and $E_{2}$ are added there will be two different circuits in the augmented graph. One readily verifies that such a configuration cannot be reduced to an exclusion graph by the removal of a single edge, hence $T$ is strongly simple. Thus we have:

THEOREM 5.1. Any two maximal trees in a connected graph are simply related.

6. Case of a finite $G$. Finally let $G$ be a finite set and take $H$ as an exclusion set with a maximal number of elements. It is then evident that a simple interchange for such a set must give another set of the same type so that in this case the assumption that $H$ is strongly simple is unnecessary. This leads to:

THEOREM 6.1. In a finite set assume that all exclusion sets with a maximal number of elements are simple. Then these sets are all simply related.

7. Crossing sets and duality (Note By T. S. Motzkin). In the sequel the forbidden sets are not assumed to be finite. 
The complement $G-H$ of an exclusion set is a crossing set $C$; it is nondisjoint from every $F$ (e.g. the selection sets appearing in the axiom of choice are special crossing sets). If $\phi$ is the family of all $F$ and $\phi^{\prime}$ the family of all $C$ then (cf. a general duality theorem in [3, p. 19]) $\phi^{\prime \prime} \supseteq \phi, \phi^{\prime \prime \prime}=\phi^{\prime}$. Here $\phi^{\prime \prime}$ is the family of all subsets of $G$ containing at least one $F$ as a subset; for if a set omits some elements in every $F$ then its complement is a crossing set. Only if $\phi$ with every $F$ includes every subset of $G$ of which an $F$ is a subset then $\phi^{\prime \prime}=\phi$ and thus there is a symmetric (and antimonotone) duality relation between such families $\phi$ and their derived families $\phi^{\prime}$.

The maximal principle for exclusion sets becomes a minimal principle for $\phi^{\prime}$. Neither $\phi$ nor $\phi^{\prime}$ need fulfill this principle. The minimal principle for $\phi$ implies (but is not implied by) the existence of a smallest $F$ within every $F$; this latter condition is equivalent to the existence of a basis (a set of members of $\phi$ none of which is a subset of any other while each $F$ contains one of these members of a subset). Neither $\phi$ nor $\phi^{\prime}$ need have a basis.

Finiteness of all members of a basis of $\phi$ implies the minimal principle for $\phi^{\prime}$. Finiteness of $\phi$ implies the existence of a basis for $\phi^{\prime}$, but not the minimal principle for $\phi^{\prime}$. The existence of a basis for $\phi$ does not imply the same for $\phi^{\prime}$; e.g. if $G$ is the set of all integers, and $\phi$ given by its basis consisting of the sets $(-k, k+1, k+2, k+3, \cdots)$, $k=0,1,2, \cdots$, then $(0,1,2, \cdots)$ is a crossing set that contains no minimal crossing set.

\section{BIBLIOGRA PHY}

1. R. L. Wilder, Introduction to the foundations of mathematics, Wiley, New YorkLondon, 1952.

2. D. König, Theorie der endlichen und unendlichen Graphen, Leipzig, 1936.

3. T. S. Motzkin, Linear inequalities, Lecture notes, University of California at Los Angeles, 1951.

YALE UNIVERSITY AND

UNIVERSITY OF CALIForNia 